\title{
Association between miR-181b and PKG 1 in myocardial hypertrophy and its clinical implications
}

\author{
WEI ZHONG ${ }^{1}$, JUN YANG ${ }^{2}$, ${\text { QIAN } \mathrm{CAO}^{3} \text { and XIAODONG HUAN }}^{1}$ \\ ${ }^{1}$ Cadre Ward; ${ }^{2}$ Department of Cardiology, Zaozhuang Municipal Hospital, Zaozhuang, Shandong 277101; \\ ${ }^{3}$ Department of Cardiology, Zaozhuang Hospital of Traditional Chinese Medicine, Zaozhuang, Shandong 277100, P.R. China
}

Received July 31, 2014; Accepted April 13, 2015

DOI: $10.3892 /$ etm.2015.2647

\begin{abstract}
The aim of this study was to explore the microRNA (miR)-181b expression in myocardial hypertrophy and to investigate its association with cGMP-dependent protein kinase type I (PKG 1) in an in vitro model. The miR-181b level in the peripheral blood was determined in patients with myocardial hypertrophy, and an in vitro model was established via phenylephrine (PE) treatment. Reverse transcription-quantitative polymerase chain reaction analysis and western blotting were performed to detect the expression levels of miR-181b, PKG 1 and hypertrophy-related genes. The results revealed that the expression of miR-181b was elevated in the peripheral blood of patients with myocardial hypertrophy, and this may have contributed to the pathology and progression of the disease. When the primary myocardial cells were treated with PE, microscopic observation and flow cytometry revealed significant hypertrophy. Furthermore, upregulation of myocardial hypertrophy-related genes, including $\beta$-myosin heavy chain, $\alpha$-sarcomeric actinin and atrial natriuretic peptide, was observed. The miR-181b expression level in the PE-treated cells was elevated, while the mRNA and protein expression levels of PKG 1 were decreased, indicating a negative correlation between miR-181b and PKG 1 in myocardial hypertrophy. In addition, when the PE-treated primary myocardial cells were transfected with miR-181b inhibitor, the reduced PKG 1 expression was restored and the myocardial hypertrophy alleviated, as indicated by the reduced cellular sizes and decreased expression levels of the myocardial hypertrophy-related genes. In conclusion, miR-181b expression has been shown to be upregulated in myocardial hypertrophy, and this may play a role in the pathogenesis of the disease by regulating the expression of PKG 1 . The present findings
\end{abstract}

Correspondence to: Dr Wei Zhong, Cadre Ward, Zaozhuang Municipal Hospital, 41 Longtou Road, Zaozhuang, Shandong 277101, P.R. China

E-mail: zhongwgx@163.com

Key words: myocardial hypertrophy, microRNA-181b,cGMP-dependent protein kinase type I, clinical implications suggest that miR-181b is a promising molecular indicator for the clinical diagnosis and treatment of cardiac hypertrophy.

\section{Introduction}

Myocardial hypertrophy, which is one of the most important risk factors for myocardial infarction and cardiac-related mortality, is a chronic condition mainly induced by long-term pressure overload (1). It has been widely accepted that cGMP-dependent protein kinase type I (PKG 1) is closely associated with the physiological function of myocardial cells. PKG 1 is an intracellular receptor that plays an important role in the activation of the BKCa channel, protein phosphorylation and the regulation of vascular smooth muscle cell gene expression. PKG 1 has also been found to inhibit the signaling pathways involved in myocardial hypertrophy (2).

MicroRNAs (miRs) are a class of non-coding 18-22 nucleotide RNA molecules, which have important roles as gene regulators in eukaryotic organisms (3). miRs bind to the 3'-untranslated region of target mRNAs and inhibit the initiation of translation. Recently, it has been shown that miRs are widely associated with pathophysiological changes in myocardial cells (4). The upregulation of miR-1 has been reported in myocardial cells, modulating the expression of myocardial connexin 43 and myocardial hypertrophy (5). Furthermore, the downregulation of miR-133 has been shown to inhibit norepinephrine- and endothelin-1-induced myocardial hypertrophy (6). In addition, the miR-181 family has recently been shown to have an important role in the pathogenesis of cancer and other diseases. The expression of miR-181b is increased in myocardial infarction, suggesting its involvement in disease progression (7). However, the mechanism underlying the involvement of miR-181b in myocardial impairment has yet to be elucidated, particularly regarding its association with PKG 1 in disease progression.

In the present study the expression of miR-181b in the peripheral blood of patients with myocardial hypertrophy was explored and its association with PKG 1 and the related mechanism was investigated in an in vitro cardiac hypertrophy model.

\section{Materials and methods}

Patients. Forty-two patients with myocardial hypertrophy (38 males and 13 females) and a mean age of 68.9 years (range, 63-71 years), who had been admitted to the 
Zaozhuang Municipal Hospital (Zaozhuang, China) between December 2011 and January 2014, were enrolled in the study. According to the clinical classification, these patients were diagnosed with stage II hypertension of a $>5$-year duration. In addition, 20 healthy subjects were used as controls. Prior written and informed consent was obtained from every participant, and the study was approved by the Ethics Review Board of the Zaozhuang Municipal Hospital.

Reverse transcription-quantitative polymerase chain reaction $(R T-q P C R)$. Total RNA was extracted using TRIzol ${ }^{\circledR}$ reagent (Invitrogen ${ }^{\mathrm{TM}}$ Life Technologies, Carlsbad,CA, USA), and the RT was conducted using the Poly(A) tailing method. qPCR was performed with the Takara SYBR ${ }^{\circledR}$ PrimeScript ${ }^{\mathrm{TM}}$ RT-PCR kit (Takara Bio, Inc., Shiga, Japan), according to the manufacturer's instructions. The reaction system contained $10 \mu \mathrm{l}$ qPCR-Mix, $0.5 \mu 1$ of each primer, $1 \mu \mathrm{l}$ cDNA and $8 \mu \mathrm{l}$ double-distilled (dd) $\mathrm{H}_{2} \mathrm{O}$. U6 was used as internal control. The primers were synthesized by Sangon Biotech (Shanghai, China), and the primer sequences were as follows: miR-181b forward, 5'-AACATTCATTGCTGTCGGT-3' (3' universal primer, which was provided in the kit, was used as the reverse primer); U6 forward, 5'-ATTGGAACGATACAGAGAAGA TTA-3' and U6 reverse, 5'-AATATGGAACGCTTCACG AAT-3'. The qPCR conditions consisted of denaturation at $95^{\circ} \mathrm{C}$ for $10 \mathrm{~min}$, followed by 40 cycles of $95^{\circ} \mathrm{C}$ for $1 \mathrm{~min}$ and $60^{\circ} \mathrm{C}$ for $30 \mathrm{sec}$. The relative expression levels of the target genes were calculated using the $2^{-\Delta \Delta C t}$ method (8).

Primary myocardial cell culture. Ventricular myocardium was separated and isolated from 3-day-old neonatal rats (Dashuo Biotechnology Co., Ltd., Chengdu, China). The tissues were digested with $5 \mathrm{ml} 0.5 \%$ trypsin and $0.5 \%$ collagenase at $37^{\circ} \mathrm{C}$ for $5 \mathrm{~min}$. The cell suspension was collected into the centrifuge tube containing $2 \mu \mathrm{l}$ calf serum (Gibco-BRL, Grand Island, NY, USA). Following centrifugation at $200 \mathrm{x}$ g for $5 \mathrm{~min}$ at room temperature, the supernatant was discarded, and the cells were re-suspended with $5 \mu 1$ HEPES-buffered Dulbecco's modified Eagle's medium (H-DMEM; Gibco-BRL) containing 10\% fetal bovine serum (FBS; Gibco-BRL). The cells were cultured in a $37^{\circ} \mathrm{C}, 5 \% \mathrm{CO}_{2}$ incubator. For the establishment of the myocardial hypertrophy model, the primary myocardial cells were subjected to treatment with $100 \mu \mathrm{m}$ phenylephrine (PE; Sigma, St. Louis, MO, USA) for $72 \mathrm{~h}$, prior to the subsequent experiments.

Immunohistochemistry. Cells were cultured on slides and then fixed with cold acetone at $4^{\circ} \mathrm{C}$ for $1 \mathrm{~h}$. Following three washes with $\mathrm{ddH}_{2} \mathrm{O}$, the slides were incubated in $\mathrm{H}_{2} \mathrm{O}_{2}$ in the dark for $20 \mathrm{~min}$, and then washed twice with phosphate-buffered saline (PBS). Sheep serum (1:200; Gibco-BRL) was added for blocking at $37^{\circ} \mathrm{C}$ for $1 \mathrm{~h}$. Rabbit anti-mouse anti- $\alpha$-sarcomeric actinin antibody $(1: 1,000$; cat. no. ab137346; Abcam, Cambridge, MA, USA) was added to the cells, which were incubated at $4^{\circ} \mathrm{C}$ overnight. Rabbit anti-mouse secondary antibody (1:200; Santa Cruz Biotechnology, Inc., Dallas, TX, USA) was then added for incubation at $37^{\circ} \mathrm{C}$ for $30 \mathrm{~min}$. DAPI (Santa Cruz Biotechnology, Inc.) was added for 5 min. Myocardial cells

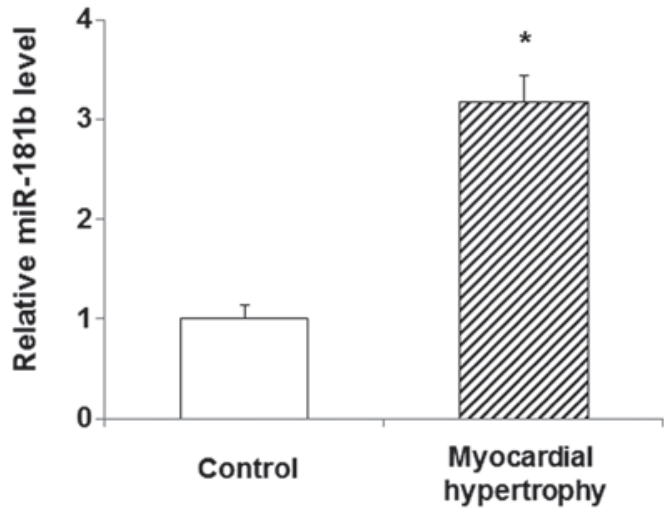

Figure 1. Increased expression level of miR-181b in the peripheral blood of patients with myocardial hypertrophy. The miR-181b level in the peripheral blood serum was determined via the quantitative polymerase chain reaction. Compared with the control group, ${ }^{*} \mathrm{P}<0.05$. miR, microRNA.

were observed using a laser scanning confocal microscope (LSM710; Carl Zeiss AG., Jena, Germany).

Western blot analysis. Cells were treated with a radioimmunoprecipitation assay buffer, and the supernatant was extracted from the lysate. Protein concentration was determined using the bicinchoninic acid assay (Pierce Biotechnology, Inc., Rockford, IL, USA), according to the manufacturer's instructions. After having been mixed with $2 \mathrm{X}$ sodium dodecyl sulfate (SDS) loading buffer, the protein samples were separated with SDS-polyacrylamide gel electrophoresis and then transferred onto a polyvinylidene difluoride membrane. The membrane was blocked using $50 \mathrm{~g} / \mathrm{l}(\mathrm{w} / \mathrm{v})$ non-fat milk at room temperature for $2 \mathrm{~h}$ and then incubated with rabbit anti-mouse anti-PKG 1 polyclonal antibody (1:1,000; cat. no. sc-211; Santa Cruz Biotechnology, Inc.) or mouse anti-GAPDH monoclonal antibody $(1: 5,000$; cat. no. sc-365062; Santa Cruz Biotechnology, Inc.) at $4^{\circ} \mathrm{C}$ overnight. The membrane was washed three times with PBS-Tween 20, and the horseradish peroxidase-conjugated goat anti-mouse immunoglobulin G (IgG) $(1: 3,000$; Santa Cruz Biotechnology, Inc.) and goat anti-rabbit $\operatorname{IgG}(1: 1,000$; Santa Cruz Biotechnology, Inc.) secondary antibodies were then incubated with the membrane, respectively, at room temperature for $2 \mathrm{~h}$.

Transfection. Transfection was performed using Lipofectamine ${ }^{\circledR} 2000$ (Invitrogen Life Technologies). Primary myocardial cells were cultured in antibiotic-free H-DMEM (Gibco-BRL) containing 10\% FBS (Gibco-BRL). Twenty-four hours later, $1.5 \mu 120 \mathrm{pmol} / \mu 1 \mathrm{miR}-181 \mathrm{~b}$ inhibitor (RiboBio Co., Ltd., Guangzhou, China) and $1 \mu \mathrm{l}$ Lipofectamine 2000 were separately mixed with $50 \mu 1$ Opti-MEM $^{\circledR}$ (Gibco-BRL). The media were then mixed and used to incubate the cells. Six hours later, the transfection medium was replaced by the H-DMEM containing $10 \%$ FBS. Random sequence was used as a transfection control.

Statistical analysis. Data are expressed as the mean \pm standard deviation. SPSS 11.0 software (SPSS Inc., Chicago, IL, USA) was used for the statistical analysis. Comparisons 
A a

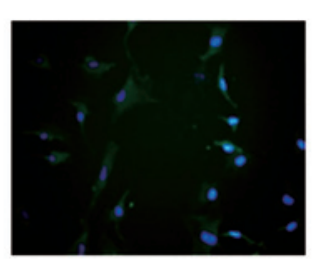

b

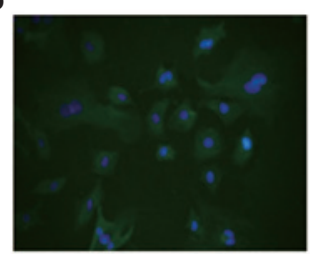

B
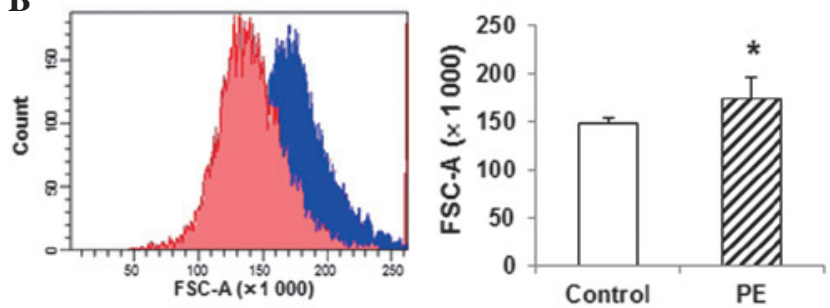

C

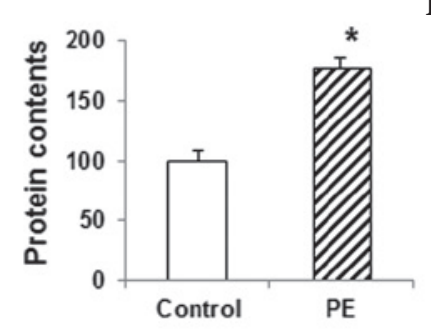

D

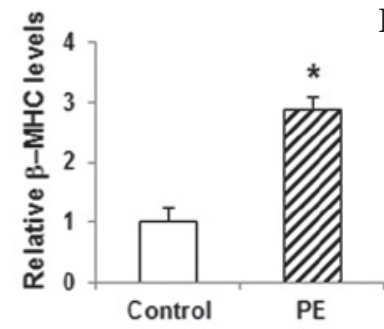

E

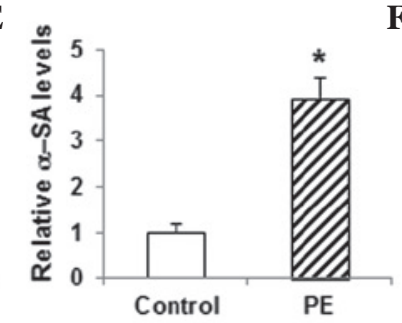

F

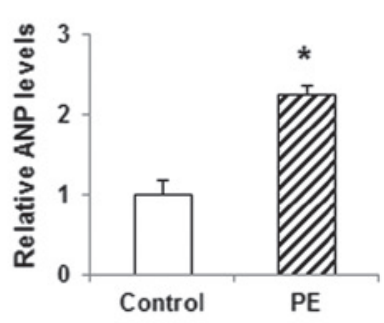

Figure 2. Construction and identification of the in vitro model of myocardial hypertrophy. (A) Primary myocardial cells from 3-day-old rats were cultured (a) with or (b) without $100 \mu \mathrm{M}$ PE (magnification, x400). Cells were stained with $\alpha$-SA antibody (green) and DAPI (blue). (B) Sizes of cardiac myocytes in the control group (red) and the PE-treated group (blue) were detected using flow cytometry. (C) Total cellular protein levels were determined using the bicinchoninic acid assay. (D-F) The mRNA expression levels of the hypertrophy-related genes (D) $\beta$-MHC, (E) $\alpha-S A$ and (F) ANP in myocardial cells were detected via the quantitative polymerase chain reaction. Compared with the control group, ${ }^{*} \mathrm{P}<0.05$. PE, phenylephrine; $\beta$-MHC, $\beta$-myosin heavy chain; $\alpha-\mathrm{SA}$, $\alpha$-sarcomeric actinin; ANP, atrial natriuretic peptide; FSC, forward scatter.

between the groups were conducted using the Student's t-test. $\mathrm{P}<0.05$ was considered to indicate a statistically significant difference.

\section{Results}

Levels of miR-181b in the peripheral blood of patients with myocardial hypertrophy. In order to explore the role of miR-181b in myocardial hypertrophy, its expression level was detected in the peripheral blood of patients diagnosed with the disease using qPCR. The results showed that the miR-181b expression in the peripheral blood of the patients was significantly increased compared with that in the blood of the normal control subjects $(\mathrm{P}<0.05)$ (Fig. 1). This elevated expression of miR-181b in myocardial hypertrophy suggests that miR-181b is involved in the pathology and progression of the disease. In the following experiments, the role of miR-181b, and its association with PKG 1 in particular, was investigated in the primary myocardial cell culture treated with PE.

Construction and identification of the in vitro model of myocardial hypertrophy. The cell model of myocardial hypertrophy was established with primary myocardial cells obtained from 3 -day-old rats. When stained with anti- $\alpha$-SA antibody and DAPI, the primary myocardial cells exhibited a fusiform morphology (Fig. 2Aa). These primary cells were subjected to PE treatment to induce hypertrophy. Following the treatment of the myocardial cells with $100 \mu \mathrm{M}$ PE for $72 \mathrm{~h}$, the immunohistochemistry results showed significant hypertrophy compared with the control group (Fig. 2Ab). Furthermore, the flow cytometric analysis showed that the size of the cardiac myocytes in the PE-treated group was significantly increased $(\mathrm{P}<0.05)$ (Fig. $2 \mathrm{~B})$. The total protein content in the PE-treated group was also significantly increased compared with that in the control group $(\mathrm{P}<0.05)$ (Fig. 2C). qPCR assays showed that the myocardial expression levels of the hypertrophy-related genes $\beta$-myosin heavy chain ( $\beta$-MHC), $\alpha$-SA and atrial natriuretic peptide (ANP) were significantly increased in the PE-treated group compared with those in the control group $(\mathrm{P}<0.05)$ (Fig. 2D-F). These results suggested that the in vitro myocardial hypertrophy model had been successfully constructed and was therefore suitable for the following analyses.

Expression levels of miR-181b and PKG 1 in myocardial hypertrophy cells. To investigate the association between miR-181b and PKG 1 in the model of myocardial hypertrophy, the expression levels of miR-181b, as well as the mRNA and protein expression levels of PKG 1, were detected using qPCR and western blot analysis. The results from the qPCR analysis showed that, compared with the control cells, the miR-181b expression was significantly elevated in the PE-treated cells $(\mathrm{P}<0.05)$ (Fig. 3A). By contrast, the mRNA level of PKG 1 was significantly decreased in the PE-treated group compared with that in the control group $(\mathrm{P}<0.05)$ (Fig. 3B). Similarly, the western blot analysis indicated that the protein expression level of PKG 1 was significantly lower in the PE-treated group than that in the control group $(\mathrm{P}<0.05)$ (Fig. 3C). These results showed that the expression level of miR-181b was elevated in the myocardial hypertrophy cells, while the mRNA and protein expression levels of PKG 1 were decreased.

Effects of miR-181b inhibition on PKG 1 expression and hypertrophy in PE-treated myocardial cells. In order to determine whether or not the changes in the expression of miR-181b and PKG 1 were associated with one another, an inhibitor of miR-181b was used to treat these myocardial hypertrophy cells and the expression level of PKG 1 was detected. The 
A

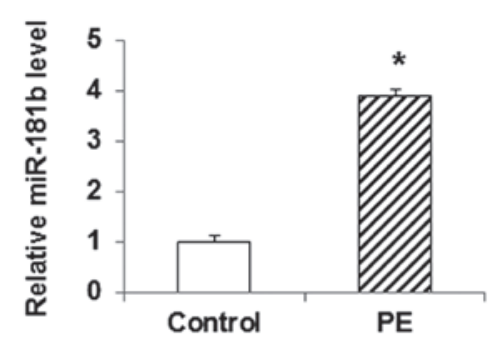

C

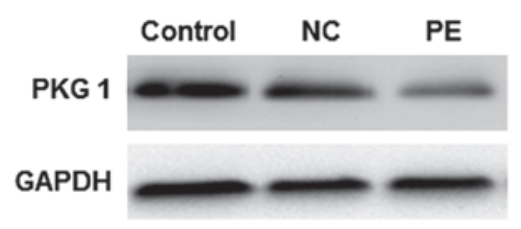

B
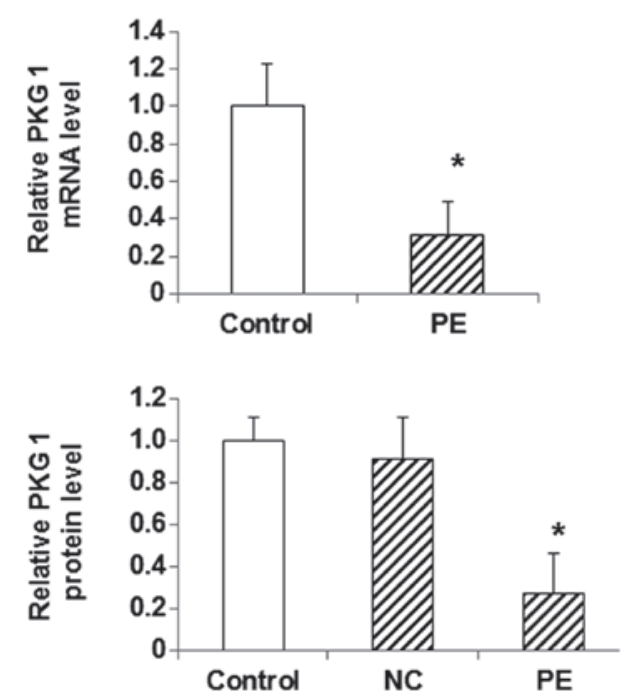

Figure 3. Expression of miR-181b and PKG 1 in myocardial hypertrophy cells. (A) The expression level of miR-181b was detected using qPCR in myocardial cells. (B) mRNA and (C) protein expression levels of PKG 1 were detected in myocardial cells using qPCR and western blotting, respectively. Compared with the control group, "P<0.05. miR, microRNA; PE, phenylephrine; NC, random sequence; PKG 1, cGMP-dependent protein kinase type I; qPCR, quantitative polymerase chain reaction.

PE-treated myocardial cells were transfected with miR-181b inhibitor, and the inhibition of miR-181b following transfection was confirmed by the results of the qPCR analysis (Fig. 4A). qPCR also showed that the mRNA expression level of PKG 1 was slightly increased following the miR-181b inhibitor transfection in PE-treated cells, compared with the control group (Fig. 4B). Furthermore, western blotting indicated that the protein expression of PKG 1 was significantly increased in the PE-treated cells following miR-181b inhibitor transfection, compared with the control cells $(\mathrm{P}<0.05)$ (Fig. 4C). Flow cytometric analysis showed a notable decrease in the myocardial cell size, a decreased total protein content and reduced mRNA expression levels of $\beta$-MHC, $\alpha-$ SA and ANP in the PE-treated myocardial cells following miR-181b inhibitor transfection $(\mathrm{P}<0.05)$ (Fig. 4D-H). These findings demonstrate the ability of miR-181b inhibition to restore the reduced PKG 1 expression in PE-treated myocardial cells and alleviate myocardial hypertrophy, as indicated by the reduced cellular sizes and decreased expression levels of myocardial hypertrophy-related genes.

\section{Discussion}

Myocardial hypertrophy has been closely linked with other chronic diseases, including hypertension, diabetes and chronic obstructive pulmonary disease (9). Long-term pressure overload is considered to be a triggering factor for myocardial hypertrophy, which can change the gene expression and signaling pathway functions as the disease progresses. When pressure overload persists, hypertrophic changes occur in myocardial cells, disturbing gene expression and protein synthesis and impairing mitochondria and endoplasmic reticulum $(10,11)$. As compensatory stress responses, protein and adenosine triphosphate synthesis and ion channel activity in these cells are subsequently enhanced (12-14), maintaining the myocardial systolic function and protecting against pressure overload; however, long-term hypertrophy could eventually result in impaired cellular structure and function. These pathological changes would in turn lead to cell death and interstitial fibrosis, eventually resulting in heart failure. The present study investigated the molecular mechanism of myocardial hypertrophy, in in order to search for novel biological indicators, which could contribute to the diagnosis and treatment of the disease.

PKG 1 has been closely associated with the physiological functions of myocardial cells, and is also involved in the modulation of $\mathrm{Ca}^{2+}$ concentration and smooth muscle tension in vessels (15). A recent study indicated that PKG 1 can inhibit myocardial cell hyperplasia and hypertrophy. Through cGMP regulation, PKG 1 can phosphorylate and activate troponin $\mathrm{T}$. In addition, PKG 1 can inhibit the calcium-dependent calcineurin/nuclear factor of activated $\mathrm{T}$ cells signaling pathway and modulate cell hypertrophy by regulating the L-type calcium channel (16); however, further studies are required to determine whether there are other mechanisms underlying the functions of PKG 1 in myocardial hypertrophy.

The role of miRNA in the pathogenesis of myocardial hypertrophy has attracted a great deal of attention in recent years. It has been reported that miR-98 and miR-9 are closely associated with the development of myocardial hypertrophy (17). The newly discovered miRNA family miR-181 has been found to promote tumor proliferation, invasion and migration (18). In this study, the role of miR-181b in the progression of myocardial hypertrophy was investigated. The results indicated that the expression level of miR-181b was significantly increased in the peripheral blood of patients with myocardial hypertrophy. To further confirm the role of miR-181b in myocardial hypertrophy, an in vitro cardiomyocyte hypertrophy model was constructed using PE induction. Following $72 \mathrm{~h}$ of PE treatment, the size and morphology of the primary myocardial cells were observed. Furthermore, the total protein content was analyzed and the expression levels of 
A
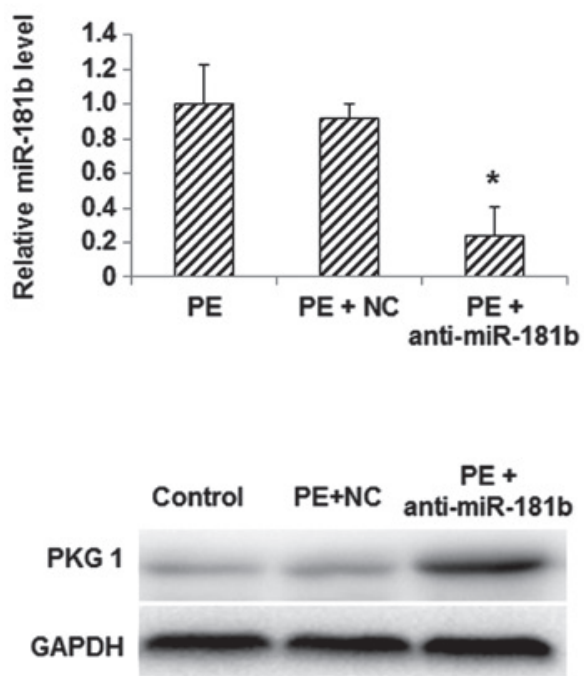

D

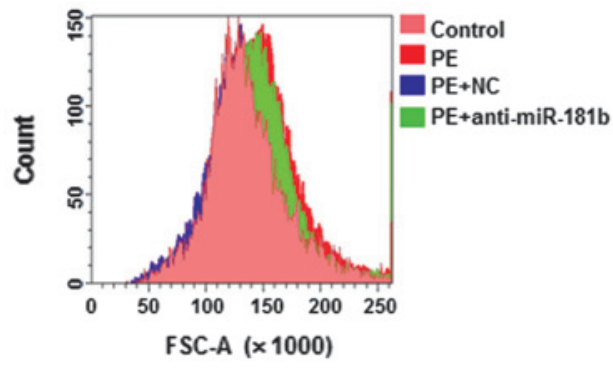

$\mathbf{E}$

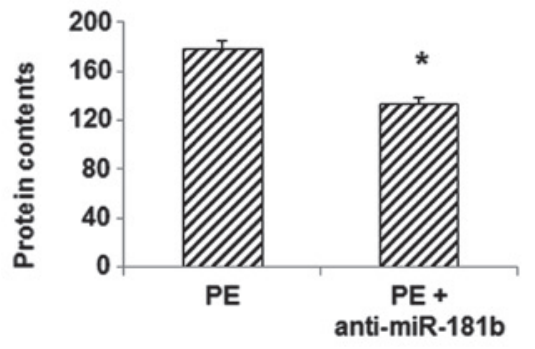

G

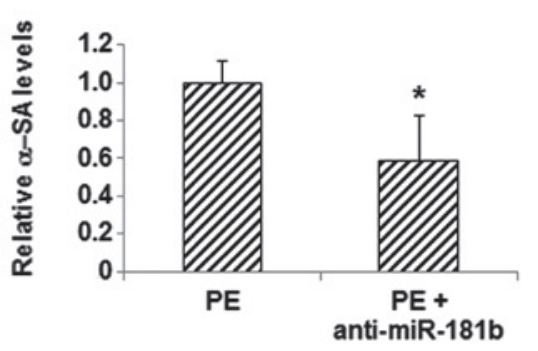

B

$\mathbf{F}$

H
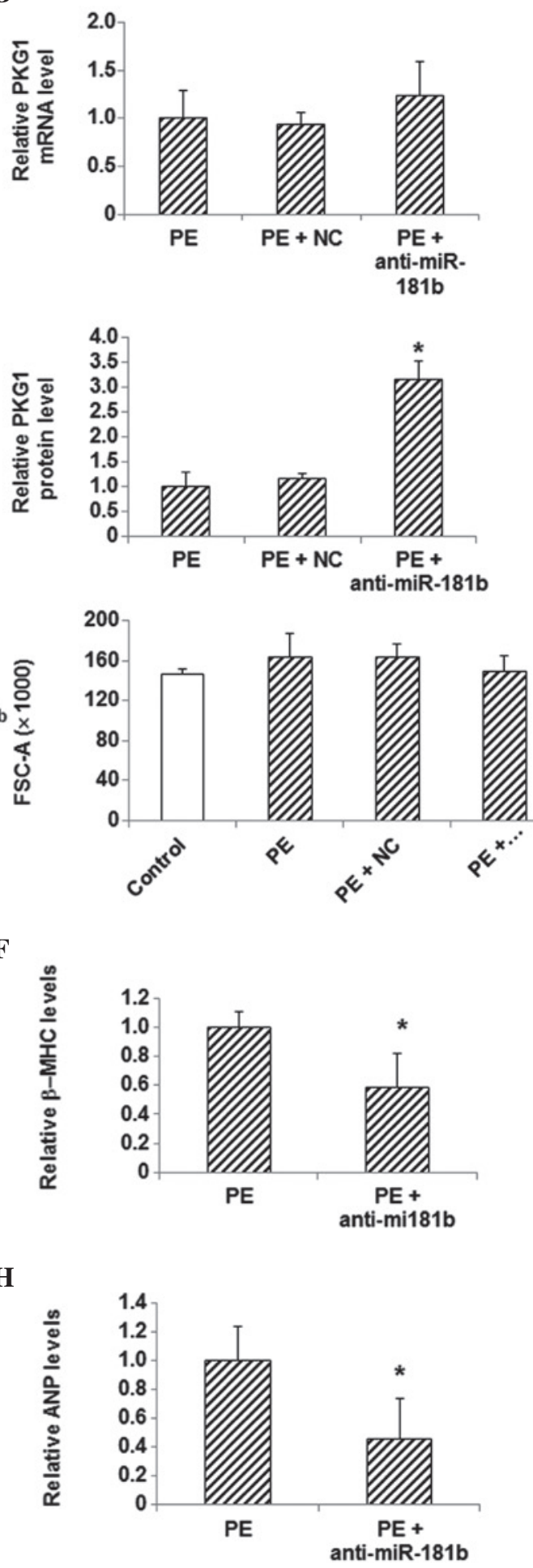

Figure 4. Effects of miR-181b inhibition on PKG 1 expression and hypertrophy in PE-treated myocardial cells. (A) The expression level of miR-181b was detected via qPCR in myocardial cells following the transfection of miR-181b inhibitor. (B) mRNA and (C) protein expression levels of PKG 1 were detected in myocardial cells using qPCR and western blotting, respectively, following miR-181b inhibitor transfection. (D) Sizes of cardiac myocytes in the control (pink), PE (red), PE + NC (green) and PE + anti-miR-181b (transfected with miR-181b inhibitor) (blue) groups were detected with flow cytometry. (E) Total cellular protein levels were determined using the bicinchoninic acid assay. (F-H) The mRNA expression levels of the hypertrophy-related genes (F) $\beta$-MHC, (G) $\alpha$-SA and (H) ANP in myocardial cells following transfection were detected using qPCR. Compared with the PE-treated group, "P<0.05. miR, microRNA; PE, phenylephrine; NC, random sequence; PKG 1, cGMP-dependent protein kinase type I; $\beta$-MHC, $\beta$-myosin heavy chain; $\alpha$-SA, $\alpha$-sarcomeric actinin; ANP, atrial natriuretic peptide; FSC, forward scatter; qPCR, quantitative polymerase chain reaction.

the myocardial hypertrophy-related genes $\beta$-MHC, $\alpha$-SA and ANP were detected. The myocardial hypertrophy model was characterized and identified according to these assessments.
The results indicated that the expression level of miR-181b was significantly increased, while the mRNA and protein expression levels of PKG 1 were decreased in the PE-treated cells, 
suggesting that miR-181b is negatively correlated with PKG 1 expression. When the PE-treated primary myocardial cells were transfected with an miR-181b inhibitor, the miR-181b expression was significantly downregulated, while the mRNA and protein expression levels of PKG 1 were markedly increased. In addition, flow cytometry showed that the size of the myocardial cells was reduced following the inhibition of miR-181b. The total cellular protein content and the expression levels of hypertrophy-related genes were significantly decreased in the PE-treated cells transfected with miR-181b inhibitor, indicating alleviated myocardial hypertrophy in these cells.

In conclusion, according to the findings of the present study, miR-181b could play an important role in the pathogenesis of myocardial hypertrophy by regulating the expression of PKG 1 . The expression level of miR-181b was significantly increased in the peripheral blood of patients with myocardial hypertrophy. In the myocardial hypertrophy model cells induced by PE treatment, the expression level of miR-181b was elevated, while the mRNA and protein expression levels of PKG 1 were decreased. When miR-181b was inhibited in these cells, the expression of PKG 1 was restored and myocardial hypertrophy was alleviated. The present results suggest that miR-181b can be used as a novel target for the clinical diagnosis and treatment of myocardial hypertrophy.

\section{Acknowledgements}

The authors would like to thank Professor Shujian Sui from the Second Hospital of Shandong University and Professor Tongbao Liu from the Shandong Provincial Hospital for their advice and assistance with the study design, sample and data collection, statistical analysis and manuscript preparation.

\section{References}

1. Oka T, Akazawa H, Naito AT and Komuro I: Angiogenesis and cardiac hypertrophy: Maintenance of cardiac function and causative roles in heart failure. Circ Res 114: 565-571, 2014.

2. Hofmann $\mathrm{F}$ and Wegener JW: cGMP-dependent protein kinases (cGK). Methods Mol Biol 1020: 17-50, 2013.
3. Wen KC, Sung PL, Yen MS, Chuang CM, Liou WS and Wang PH: MicroRNAs regulate several functions of normal tissues and malignancies. Taiwan J Obstet Gynecol 52: 465-469, 2013.

4. Hodgkinson CP, Kang MH, Dal-Pra S, Mirotsou M and Dzau VJ: MicroRNAs and Cardiac Regeneration. Circ Res 116: 1700-1711, 2015.

5. Wu Y, Ma XJ, Wang HJ, et al: Expression of Cx43-related microRNAs in patients with tetralogy of Fallot. World J Pediatr 10: 138-144, 2014.

6. Katta A, Thulluri S, Manne ND, et al: Overload induced heat shock proteins (HSPs), MAPK and miRNA (miR-1 and miR133a) response in insulin-resistant skeletal muscle. Cell Physiol Biochem 31: 219-229, 2013.

7. Domigan CK and Iruela-Arispe ML: Recent advances in vascular development. Curr Opin Hematol 19: 176-183, 2012.

8. Bubner B and Baldwin IT: Use of real-time PCR for determining copy number and zygosity in transgenic plants. Plant Cell Rep 23: 263-271, 2004.

9. Lyon RC,ZanellaF,Omens JH and Sheikh F: Mechanotransduction in cardiac hypertrophy and failure. Circ Res 116: 1462-1476, 2015.

10. Novoyatleva T, Sajjad A and Engel FB: TWEAK-Fn14 cytokine-receptor axis: A new player of myocardial remodeling and cardiac failure. Front Immunol 5: 50, 2014.

11. Guan HS, Shangguan HJ, Shang Z, Yang L, Meng XM and Qiao SB: Endoplasmic reticulum stress caused by left ventricular hypertrophy in rats: Effects of telmisartan. Am J Med Sci 342: 318-323, 2011.

12. Soares JB, Rocha-Sousa A, Castro-Chaves P, Henriques-Coelho T and Leite-Moreira AF: Inotropic and lusitropic effects of ghrelin and their modulation by the endocardial endothelium, NO, prostaglandins, GHS-R la and KCa channels. Peptides 27: 1616-1623, 2006.

13. Tagashira H, Bhuiyan MS, Shioda N and Fukunaga K: Fluvoxamine rescues mitochondrial $\mathrm{Ca}^{2+}$ transport and ATP production through $\sigma(1)$-receptor in hypertrophic cardiomyocytes. Life Sci 95: 89-100, 2014

14. Ghule AE, Kulkarni CP, Bodhankar SL and Pandit VA: Effect of pretreatment with coenzyme Q10 on isoproterenol-induced cardiotoxicity and cardiac hypertrophy in rats. Curr Ther Res Clin Exp 70: 460-471, 2009.

15. Yang HM, Kim BK, Kim JY, et al: PPAR $\gamma$ modulates vascular smooth muscle cell phenotype via a protein kinase G-dependent pathway and reduces neointimal hyperplasia after vascular injury. Exp Mol Med 45: e65, 2013.

16. Vandael DH, Mahapatra S, Calorio C, Marcantoni A and Carbone E: Cav1.3 and Cav1.2 channels of adrenal chromaffin cells: Emerging views on cAMP/cGMP-mediated phosphorylation and role in pacemaking. Biochim Biophys Acta 1828: 1608-1618, 2013

17. Harada M, Luo X, Murohara T, Yang B, Dobrev D and Nattel S: MicroRNA regulation and cardiac calcium signaling: Role in cardiac disease and therapeutic potential. Circ Res 114: 689-705, 2014.

18. Tong SJ, Liu J, Wang X and Qu LX: microRNA-181 promotes prostate cancer cell proliferation by regulating DAX-1 expression. Exp Ther Med 8: 1296-1300, 2014. 